\title{
Consumo de alcohol y violencia escolar en adolescentes escolarizados
}

\author{
P.S.S.L.E Miguel Ángel Hernández López \\ m.angel.hernandez.lopez@gmail.com \\ Universidad Juárez Autónoma de Tabasco \\ P.S.S.L.E María Isabel García Cupil \\ isagarcup@hotmail.com \\ Universidad Juárez Autónoma de Tabasco
}

M.E José Juan López Cocotle *

Juan.lopezc@ujat.mx

https://orcid.org/0000-0002-0919-2630

Universidad Juárez Autónoma de Tabasco

M.E Pedro González Angulo

pedrogonzalez8203@gmail.com

https://orcid.org/0000-0001-6098-1945

Universidad Juárez Autónoma de Tabasco

Dr. Manuel Antonio López Cisneros

mlcisneros@hotmail.com

https://orcid.org/0000-0002-9384-5752

Universidad Autónoma del Carmen

Dr. Javier Salazar Mendoza

jasalazar@uv.mx

https://orcid.org/0000-0001-9172-8731

Universidad Veracruzana

\section{RESUMEN}

Objetivo: conocer la relación entre el consumo de alcohol y violencia escolar en adolescentes escolarizados. Método: estudio cuantitativo, descriptivo y correlacional en una muestra de 222 estudiantes de secundaria, se utilizó muestreo probabilístico estratificado. Los instrumentos utilizados fueron: Cuestionario de Identificación de Desórdenes por Uso de Alcohol (AUDIT) y Escala de Conducta Delictiva y Violenta en el aula (ECV). Los datos fueron procesados en el programa SPSS versión 22. Se utilizó coeficiente de correlación de Spearman y la prueba H de Kruskal Wallis. Resultados: se encontró una correlación positiva entre el consumo de alcohol y la violencia escolar $(p<.001)$. Los patrones de consumo de alcohol mostraron diferencias significativas con la violencia escolar, conducta violenta y victimización $(p=.006, p=.003$ y $p=0.43$, 
respectivamente). Conclusión: existe asociación entre el consumo de alcohol y la violencia escolar en los adolescentes de secundaria, es preciso atender a esta población, debido a su vulnerabilidad de presentar conductas adictivas que se atribuye a conductas violentas en las aulas.

Palabras clave: Consumo de alcohol; Consumo en menores de edad; Violencia; Adolescente; Educación. 


\title{
Alcohol consumption and school violence in school adolescents
}

\begin{abstract}
Objective: to know the relationship between alcohol consumption and school violence in school adolescents. Method: quantitative, descriptive and correlational study in a sample of 222 high school students, using stratified probability sampling. The instruments used were: Alcohol Use Disorder Identification Questionnaire (AUDIT) and Crime and Violence in the Classroom Scale (CCS). The data were processed in the SPSS version 22 program. Spearman's correlation coefficient and Kruskal Wallis' H-test were used. Results: A positive correlation was found between alcohol consumption and school violence $(\mathrm{p}<.001)$. Drinking patterns showed significant differences with school violence, violent behavior, and victimization $(\mathrm{p}=.006, \mathrm{p}=.003$, and $\mathrm{p}=0.43$, respectively). Conclusion: there is an association between alcohol consumption and school violence in secondary school adolescents. This population needs to be addressed, due to its vulnerability to presenting addictive behaviors that are attributed to violent behavior in the classroom.
\end{abstract}

Keywords: Alcohol drinking; Underage drinking; Violence; Adolescent; Education

Artículo recibido: 05 de Abril 2021 Aceptado para publicación: 28 de Mayo 2021

Correspondencia: juan.lopezc@ujat.mx Conflictos de Interés: Ninguna que declarar 


\section{INTRODUCCIÓN}

El consumo de bebidas alcohólicas es un grave problema de salud pública en México que requiere de acciones preventivas inmediatas y de promoción de la salud. En la actualidad, el consumo de alcohol es considerado una práctica socialmente aceptada y se le reconoce como vehículo de socialización en diversos grupos sociales; uno de ellos, los adolescentes. Entre los principales efectos y repercusiones que provoca el consumo de alcohol se encuentran violencia, homicidios y accidentes automovilísticos (Tood, 2010; Ahumada, Gámez, \& Valdez, 2017).

La Organización Mundial de la salud (OMS, 2018), define al alcohol, como una sustancia psicoactiva con propiedades causantes de dependencia. El consumo nocivo, conlleva una pesada carga social y económica para las sociedades. En el mundo, 320,000 adolescentes entre 15 y 29 años mueren por causas relacionadas con el alcohol, representando un 9\% de la mortalidad de este grupo etario. En México, es la cuarta causa de muerte de la población con el 8.4\% (Ahumada et al., 2017).

Existen diferentes factores sociales que influyen de forma directa en el consumo de alcohol por los adolescentes, como la búsqueda de compañía en fiestas o reuniones, donde la justificación es convivir con los amigos, poca supervisión por parte de los padres y un elevado nivel de rechazo percibido. Con respecto al motivo y sensaciones percibidas por el adolescente al consumir alcohol, se ha observado que produce placer y es agradable el sabor de la bebida, además de que buscan experimentar y saber lo que se siente probar algo nuevo (Morales, Cabrera, Pérez, \& Amaro, 2015).

El consumo nocivo de alcohol entre los adolescentes cada vez es más preocupante, ya que ocasiona la reducción del autocontrol y aumenta los comportamientos de riesgo; entre ellos, generación de violencia, relaciones sexuales no protegidas y puede provocar problemas de salud en etapas posteriores de la vida e influir en la esperanza de vida (OMS, 2017).

A nivel nacional, el consumo de alcohol diario, según resultados del ENCODAT 20162017, reflejan que, la población de 12 a 65 años se encuentra en el 2.9\%, siendo mayor el porcentaje en los hombres de 18 a 65 años, alcanzando el 5\%, en tanto que las mujeres en este rango de edad están en el 1.2\%. La edad de inicio en población de 12 a 65 años a nivel nacional, en hombres es entre 17 años y menos (61.5\%); en las mujeres entre los 18 y 25 años (47.1\%) (Centros de Integración Juvenil [CIJ], 2018). 
En este mismo tenor, en el estado de Tabasco, el consumo diario de alcohol es del 3.5\%, en hombres de 18 a 65 años el mayor porcentaje que es de 5.9\%, en mujeres de 12 a 17 años, el porcentaje es menor que los hombres en este rango de edad con un $1.6 \%$ en mujeres y un 4.4\% en hombres; las mujeres, de 12 a 17 años, tiene un porcentaje en este consumo de $2.8 \%$ en comparación a los hombres que tienen el 4.0\% (CIJ, 2018).

El consumo de alcohol, puede ser un factor importante para presentar conductas problemáticas entre las que se encuentra la violencia escolar, algunos estudios han relacionado la violencia con el consumo de alcohol (Aguilera, Muñoz \& Orozco, 2007; Rivero, Barona \& Petriz, 2011). La violencia en el ámbito escolar es una realidad que bloquea cada día a millones de niños y jóvenes el derecho humano fundamental de la educación. Una prioridad estratégica de la Organización de las Naciones Unidas para la Educación, la Ciencia y la Cultura (UNESCO, 2014), consiste en garantizar que todos los niños y jóvenes tengan acceso a ambientes de aprendizaje seguro, inclusivo y sano.

Cabe destacar que, México se posiciona como primer lugar en violencia escolar a nivel internacional, afectando acerca de 18 millones 782 mil estudiantes, los cuales son víctimas de acoso, insultos o amenazas con armas, violencia física, fotografías o videos vergonzosos difundidos a través de las redes sociales. Respecto a esto, la Dirección General de Prevención del Delito de la Procuraduría General de la República, en México, señaló en el 2011, que uno de cada seis casos de victimización y acoso escolar terminaría en suicidio o intento de suicidio (Gardea, López, Alonso, Castillo, \& Alonzo, 2015; Ayala, 2015).

En la revisión de la literatura se han identificado pocos estudios sobre el consumo de alcohol y su relación con la violencia escolar, de estos, la mayoría enfocados en población del nivel medio superior, poco se ha explorado este fenómeno en adolescentes de secundaria, por lo tanto, este estudio se planteó como objetivo: conocer la relación entre consumo de alcohol y la violencia escolar en adolescentes escolarizados.

\section{MATERIALES Y MÉTODOS}

Estudio de tipo cuantitativo con diseño descriptivo y correlacional. La población estuvo representada por 520 adolescentes inscritos en una institución de educación secundaria, turno vespertino, del estado de Tabasco, México. La muestra se determinó con base en la fórmula población finitas o conocidas (Cochran, 2000), quedó conformada por 222 
adolescentes. El muestreo fue de tipo probabilístico estratificado por afijación igual y aleatorio simple para cada estrato (Burns \& Grove, 2004; Polit \& Hungler, 2000).

Se incluyeron a los adolescentes que aceptaron participar voluntariamente y aquellos que contaran con el asentimiento firmado por sus padres y/o tutores. Se excluyeron a los que no asistieron el día de la aplicación de los instrumentos. Para la recolección de los datos se utilizaron 3 instrumentos: una Cédula de Datos Sociodemográficos con los siguientes rubros: edad, sexo, grado escolar y actividades del adolescente en su tiempo libre y convivencia familiar. El segundo instrumento fue el Cuestionario de Identificación de Desórdenes por Uso de Alcohol (AUDIT), que tiene como objetivo medir el consumo de alcohol y la detección temprana de personas con problemas de consumo, consta de 10 preguntas que clasifican el consumo de alcohol en patrones de consumo; de 0 a 7 puntos consumo de bajo riesgo, consumo de riesgo de 8 a 15 puntos, perjudicial de 16 a 19 puntos y consumo dependiente puntuaciones mayores a 20 puntos. Los reactivos cuentan con una serie de respuestas escala tipo Likert y una escala de puntuación de cero a cuatro, la escala total tiene una puntuación mínima de cero y máxima de 40 , cuenta con una consistencia interna por Alpha de Cronbach de 0.80 (Babor, Higgins, Saunders, \& Monteiro, 2001; Tegoma \& Cortaza, 2016).

El tercer instrumento utilizado fue la Escala de Conducta Delictiva y Violenta en el Aula, elaborada por Rubini y Pombeni en 1992, la cual evalúa la participación en comportamientos de violencia, se consideran tanto los delitos manifiestos (ej. robar dinero u objetos de valor) como la trasgresión de normas sociales o reglamentos escolares (ej. hacer graffiti en las paredes del instituto). La versión en español, fue adaptada por el grupo de investigación LISIS de la Universidad de Valencia, el instrumento está compuesto por 19 ítems (escala 1-5) divididos en dos subescalas. Las opciones de respuestas van de nunca a muchas veces, las primeras 13 preguntas miden la conducta violenta y los seis restantes miden la victimización. La escala global de la adaptación española reporta un Alpha de Cronbach de .87. El puntaje mínimo es de 19 y el máximo de 95 (Vera \& Gálvez, 2014), esta versión se ha aplicado con éxito en muestras de población mexicana (Villareal, 2009; Bonilla, 2016).

Para la recolección de datos, se visitó a la institución elegida para el desarrollo del estudio solicitando la autorización para la ejecución del proyecto a las autoridades correspondientes, una vez aprobada la solicitud, se contó con la lista de asistencia (marco 
muestral) de cada grado y grupo, se seleccionaron aleatoriamente a 74 adolescentes de cada grado $\left(1^{\circ}, 2^{\circ}\right.$ y $\left.3^{\circ}\right)$ y 24 adolescentes de cada grupo (A, B y C). A los seleccionados, se les explicó el propósito del estudio y les fue entregado el asentimiento informado para padres y/o tutores. El día de la aplicación de los instrumentos, se pidió entregarán el asentimiento firmado por sus padres para poder participar, aquellos que cumplieron con este requisito y aceptaron participar voluntariamente firmaron consentimiento informado. Se resolvieron dudas y se dieron instrucciones precisas para responder correctamente los cuestionarios.

Los datos fueron procesados a través del paquete estadístico Statistical Package for the Social Sciences (SPSS) versión 22 para Windows mediante frecuencias y proporciones, para conocer la distribución de los datos se utilizó la prueba Kolmogorov-Smirnov con corrección de Lilliefors misma que determinó el uso de estadística no paramétrica. Con el fin de medir la fuerza y la dirección de la asociación entre las variables en estudio, se hizo uso del coeficiente de correlación de Spearman (Díaz, García, León, Ruíz \& Francisca, 2014). El presente estudio se realizó conforme a lo dispuesto en el Reglamento de la Ley General de Salud en materia de Investigación para la Salud (Diario Oficial de la Federación [DOF], 2014).

\section{RESULTADOS Y DISCUSIÓN}

La tabla 1 muestra que, del total de los participantes, el 52.3\% (116) son del sexo femenino y el 47.7\% (106) del sexo masculino. También se observa que el 34.2\% (76) cuenta con 14 años de edad. La mayoría de los participantes se encuentra cursando el segundo grado $40.1 \%$ (89), el $28.4 \%$ (63) solo estudia y el $69.4 \%$ (154) viven con sus padres.

Tabla 1. Datos sociodemográficos

\begin{tabular}{|c|c|c|}
\hline Características & $f$ & $\%$ \\
\hline \multicolumn{3}{|l|}{ Sexo } \\
\hline Femenino & 116 & 52.3 \\
\hline Masculino & 106 & 47.7 \\
\hline \multicolumn{3}{|l|}{ Edad } \\
\hline 12 & 54 & 24.3 \\
\hline 13 & 70 & 31.5 \\
\hline 14 & 76 & 34.2 \\
\hline 15 & 22 & 10.0 \\
\hline
\end{tabular}




\begin{tabular}{lcc}
\hline Grado escolar & & \\
Primero & 67 & 30.2 \\
Segundo & 89 & 40.1 \\
Tercero & 66 & 29.7 \\
¿A qué dedicas tu tiempo libre? & & \\
Trabajar & 13 & 5.8 \\
Estudiar & 63 & 28.4 \\
Estudiar y trabajar & 31 & 14.0 \\
Jugar & 90 & 40.5 \\
Otro & 25 & 11.3 \\
¿Con quién vives? & & \\
Padre & 154 & 69.4 \\
Solo madre & 35 & 15.7 \\
Solo padre & 7 & 3.2 \\
Abuelos & 19 & 8.6 \\
Solo & 1 & 0.5 \\
Otros & 6 & 2.6 \\
\hline
\end{tabular}

Nota. Elaboración propia.

$$
f=\text { Frecuencia. } \%=\text { Porcentaje. } \mathrm{n}=222
$$

En la tabla 2, se observan los patrones de consumo de alcohol. El $91 \%$ presenta un consumo de bajo riesgo, seguido de un consumo de riesgo (5.9\%), solo el $1.8 \%$ un consumo perjudicial.

Tabla 2. Patrones de consumo de alcohol

\begin{tabular}{lcc}
\hline \multicolumn{1}{c}{ Patrones de consumo } & $\boldsymbol{f}$ & \% \\
\hline Consumo de bajo riesgo & 202 & 91 \\
Consumo de riesgo & 13 & 5.9 \\
Consumo perjudicial & 3 & 1.4 \\
Consumo dependiente & 4 & 1.8 \\
\hline
\end{tabular}

Nota. Elaboración propia.

$f=$ Frecuencia. $\%=$ Porcentaje. $\mathrm{n}=222$

Respecto a la Escala de Conducta Violenta, se encontró que, el comportamiento de violencia más frecuente entre los adolescentes fue insultar a compañeros(as) de clase ( $M$ $=1.6, D E=1)$, seguido de agredir y pegar a los compañeros de la escuela $(M=1.3, D E$ $=.63$ ), en la escala de victimización, el comportamiento más frecuente reportado por los adolescentes fue alguien me miró con mala cara $(M=1.9, D E=1.2)$, tabla 3 . 
Tabla 3. Escala de conducta delictiva y violenta en el aula

\begin{tabular}{|c|c|c|c|c|}
\hline Comportamiento de violencia & $M$ & $D E$ & Mín & Máx \\
\hline \multicolumn{5}{|l|}{ Subescala de Conducta violenta } \\
\hline He pintado o dañado las paredes de la escuela. & 1.3 & .74 & 1 & 5 \\
\hline He robado objetos de mis compañeros o de la escuela. & 1.1 & .46 & 1 & 5 \\
\hline He insultado o engañado a propósito a los profesores. & 1.3 & .85 & 1 & 5 \\
\hline He dañado el carro de los profesores. & 1.0 & .38 & 1 & 4 \\
\hline $\begin{array}{l}\text { He hecho equivocarse a un/a compañero/a de clase en } \\
\text { las tareas a propósito. }\end{array}$ & 1.3 & .85 & 1 & 5 \\
\hline He agredido y pegado a los compañeros de la escuela. & 1.3 & .63 & 1 & 4 \\
\hline He molestado o fastidiado al profesor/a en clase. & 1.3 & .75 & 1 & 5 \\
\hline He roto los cristales de las ventanas de la escuela. & 1.1 & .47 & 1 & 4 \\
\hline He insultado a compañeros/as de clase. & 1.6 & 1.0 & 1 & 5 \\
\hline He provocado conflictos y problemas en clase. & 1.2 & .59 & 1 & 5 \\
\hline He respondido agresivamente a mis profesores/as. & 1.1 & .53 & 1 & 5 \\
\hline He roto apuntes y trabajos de mis compañeros/as. & 1.1 & .58 & 1 & 5 \\
\hline He provocado problemas entre mis compañeros/as. & 1.2 & .59 & 1 & 5 \\
\hline \multicolumn{5}{|l|}{ Subescala de Victimización } \\
\hline Alguien de la escuela me miró con mala cara & 1.9 & 1.2 & 1 & 5 \\
\hline Algún compañero me insultó o me pegó & 1.5 & .96 & 1 & 5 \\
\hline Algún compañero me robo algo & 1.6 & 1.1 & 1 & 5 \\
\hline Se burlaron de mí en clase o me hicieron daño & 1.3 & .89 & 1 & 5 \\
\hline Alguien de la escuela ofendió a mi familia & 1.4 & .92 & 1 & 5 \\
\hline $\begin{array}{l}\text { Alguien de la escuela me echo la culpa de algo que yo } \\
\text { no había hecho. }\end{array}$ & 1.6 & 1.1 & 1 & 5 \\
\hline
\end{tabular}

Nota. Elaboración propia. $M=$ Media. $D E=$ Desviación estándar. $\mathrm{n}=222$

En la tabla 4, se observa que existe correlación positiva entre el consumo de alcohol y la Escala de Conducta Delictiva y Violenta en el aula $(p<.001)$, y sus correspondientes sub escalas; conducta violenta $(p<.001)$ y victimización $(p=.002)$. Los patrones de consumo mostraron diferencias estadísticamente significativas con la ECV, conducta violenta y victimización ( $p=.006, p=.003$ y $p=0.43$, respectivamente), las medias más altas se encontraron en el consumo dependiente (tabla 5). 
Tabla 4. Consumo de alcohol y violencia escolar

\begin{tabular}{lrcc}
\hline & ECV Global & Conducta violenta & Victimización \\
\hline AUDIT & $.278^{* *}$ & $.297 * *$ & $.211^{* *}$
\end{tabular}

$\overline{\text { Nota. } A U D I T}=$ Cuestionario de Identificación de Desórdenes por Uso de Alcohol. ECV $=$ Escala de conducta delictiva y violenta en el Aula. $* *<.01$

Tabla 5. Patrones de consumo de alcohol y violencia escolar

\begin{tabular}{|c|c|c|c|c|c|c|c|c|c|c|}
\hline \multirow{3}{*}{$\begin{array}{c}\text { Violencia } \\
\text { escolar }\end{array}$} & \multicolumn{8}{|c|}{ Patrones de consumo de alcohol } & \multirow{3}{*}{$\boldsymbol{H}$} & \multirow{3}{*}{$p$} \\
\hline & \multicolumn{2}{|c|}{$\begin{array}{c}\text { Bajo } \\
\text { riesgo }\end{array}$} & \multicolumn{2}{|c|}{ Riesgo } & \multicolumn{2}{|c|}{ Perjudicial } & \multicolumn{2}{|c|}{ Dependiente } & & \\
\hline & $M$ & $D E$ & $M$ & $D E$ & $M$ & $D E$ & $M$ & $D E$ & & \\
\hline ECV global & 25.0 & 7.8 & 28 & 9.1 & 45.3 & 24.3 & 47.2 & 14.9 & 12.3 & .006 \\
\hline Conducta & 15.7 & 4.1 & 17.9 & 6.2 & 29.6 & 15.2 & 31 & 10.5 & 13.7 & .003 \\
\hline \multicolumn{11}{|l|}{ violenta } \\
\hline Victimización & 9.3 & 4.6 & 10.0 & 3.8 & 15.6 & 9 & 16.2 & 7.5 & 8.1 & .043 \\
\hline
\end{tabular}

Nota. $E C V=$ Escala de conducta delictiva y violenta en el Aula. $M=$ media. $D E=$ desviación estándar. $H=$ estadístico de Kruskal Wallis. $p<.05 . \mathrm{n}=222$

De acuerdo a los hallazgos del presente estudio, se encontró mayoritariamente un consumo de bajo riesgo (91\%), seguido de un consumo de riesgo (5.9\%), esto difiere con lo reportado por Ulloa (2014), donde se encontró que una cuarta parte (25\%) de los participantes muestran un consumo sensato, el 58.5\% tiene un consumo dependiente y el 57.9\% un consumo perjudicial. De igual manera, Telumbre y Sánchez (2015), reportan un predominio en el consumo dañino en el 38\% de los adolescentes, seguido por un consumo sensato de alcohol (32.2\%) y el $24.8 \%$ de consumo perjudicial, asimismo, Pulido (2014) reporta que los estudiantes tienen un mayor consumo dependiente (31\%), $12.6 \%$ consumo sensato y $22,4 \%$ consumo perjudicial. Una de las principales razones por la cual se pueda presentar esta situación es por distribución geográfica donde se realizó el estudio y que existe mayor muestra encuestada, considerar también el contexto de desarrollo de los adolescentes.

En cuanto a la conducta violenta se encontró que las más frecuentes fueron insultar a compañeros en clase, seguido de hacer equivocar a un (a) compañero (a) de clase en las tareas a propósito y agredir y pegar a compañeros de la escuela, en victimización se destaca; que alguien de la escuela los mira con mala cara, alguien les echo la culpa de 
algo que no habían hecho y algún compañero los insulta o pega, estos hallazgos fueron similares a lo reportado por Pulido (2014), donde destaca que las conductas violentas más frecuentes fueron insultos a compañeros de clase (agresiones físicas y verbales) e insultos y engaños a profesores. Respecto a indicadores de victimización, los indicadores más frecuentes en estudiantes fueron, que perciben que alguien los mira con mala cara, los han culpado de algo que no han realizado, entre otros. Estas similitudes permiten inferir que la violencia escolar se presenta en la vida escolar del adolescente.

Ulloa (2014) destaca que el consumo de alcohol se relacionó positivamente con la violencia escolar y con la victimización en estudiantes de bachillerato, Gardea et al. (2015) menciona que la violencia escolar y victimización tiene relación significativa con el consumo de alcohol. Los hallazgos reportados por Flores, López, Guzmán, Rodríguez y Jiménez (2019) muestra relación entre el consumo de alcohol y la agresividad en adolescente de secundaria. Lo anterior, coincide con lo encontrado en este estudio, donde se reporta una correlación positiva entre el consumo de alcohol (AUDIT) con las variables; conducta violenta y victimización. Esto demuestra que el consumo de alcohol en los adolescentes, en este caso de educación secundaria, tiene impacto sobre la conducta violenta y está presente en este tipo de población escolar.

\section{CONCLUSIÓN}

Los estudiantes de educación secundaria presentan, en su mayoría, un consumo de alcohol de bajo riesgo, solo un mínimo porcentaje lo hace de manera dependiente. Recordemos que la adolescencia es la etapa de mayor vulnerabilidad para que se inicie el consumo de alcohol y que éste, sea sensato, dependiente o dañino, pueda convertirse en una conducta adictiva. La violencia escolar está presente también durante esta etapa de vida en las aulas, la cual se refleja, principalmente mediante insultos y agresiones físicas hacías otros compañeros, dentro de las acciones que provocan que los estudiantes sean violentos con sus pares, se concentran en sentirse agredidos visualmente, por ser víctimas de robo y ser culpados de acciones no realizados por ellos. La violencia escolar en el aula se puede atribuye al consumo de alcohol, ya que estas dos variables presentan una asociación positiva estadísticamente significativa.

Dentro de las limitaciones encontradas en el estudio, fue que solo se estudió un turno escolar (vespertino) y de una sola institución, también es importante mencionar que los participantes, aunque se les garantizó la confidencialidad de los datos, pudieron no 
responder con veracidad por temor a alguna represalia por parte de sus padres y/o tutores o por la institución. Se recomienda estudios con muestras más amplias y conviene sumar a los docentes y padres para poder comparar los hallazgos que se pudieran encontrar respecto a la conducta del adolescente dentro de las aulas y en los hogares.

\section{LISTA DE REFERENCIAS}

Aguilera, G. M., Muñoz, A. G. \& Orozco, M. A. (2007). Disciplina, violencia y consumo de sustancias nocivas a la salud en escuelas primarias y secundarias de México. Ciudad de México: Instituto Nacional Para la Evaluación de la Educación. Recuperado de https://www.inee.edu.mx/wpcontent/uploads/2019/01/P1D230.pdf

Ahumada, J. G., Gámez, M. E. \& Valdez C. (2017). El consumo de alcohol como problema de salud pública. México. Ra Ximhai, 2(13), 13-24. Recuperado de https://www.redalyc.org/pdf/461/46154510001.pdf

Ayala, C. M. R. (2015). Violencia escolar: un problema complejo. Ra Ximhai, 11(4): 493509. Recuperado de https://www.redalyc.org/pdf/461/46142596036.pdf

Babor, T. F., Higgins, J. C., Saunders, J. B. \& Monteiro, M. G. (2001). AUDIT Cuestionario de Identificación de los Trastornos debidos al Consumo de Alcohol. Organización Mundial de la Salud. Recuperado de https://www.who.int/substance_abuse/activities/en/AUDITmanualSpanish.pdf

Bonilla, C. C. E. (2016). La dinámica de la violencia escolar y su relación con la interacción familiar: una mirada desde los adolescentes escolarizados. Redes. 33(2016): 53-66. Recuperado de http://redesdigital.com.mx/index.php/redes/article/view/67/125

Burns. N, \& Grove, S. K. (2004). Investigación en Enfermería. Madrid: Elsevier Saunder Centro de Integración Juvenil, A.C (2018). Diagnóstico del consumo de drogas del área de influencia del cij Villahermosa. Recuperado de http://www.cij.gob.mx/ebco20182024/9180/9180CD.html\#: :text=Por\%20otro\%20lado\%2C\%20a\%20nivel,que $\% 2010$ \% 20 hombres\%20en\%20este

Cochran, W. (2000). Técnicas de muestreo. México: Ed. Continental

Diario Oficial de la Federación. (2014). DECRETO por el que se reforman, adicionan y derogan diversas disposiciones del Reglamento de la Ley General de Salud en 
Materia de Investigación para la Salud. Recuperado de https://www.dof.gob.mx/nota_detalle.php?codigo $=5339162 \&$ fecha $=02 / 04 / 201$ $\underline{4}$

Díaz, I., García, C., León, M., Ruíz, F. \& Torres, F. (2014). Guía de Asociación entre variables (Pearson y Spearman en SPSS). Recuperado de https://www.ucursos.cl/facso/2014/2/SO01007/1/material_docente/bajar?id_material=994690

Flores, G. P.L., López, G. K. S., Guzmán, F. F. R., Rodríguez, A. L., \& Jiménez, P. B. I. (2019). Consumo de alcohol y su relación con la agresividad en adolescentes de secundaria. Revista Internacional De Investigación En Adicciones, 5(1), 31-38. https://doi.org/10.28931/riiad.2019.1.05

Gardea, D., López, K. S., Alonso, B. A., Castillo M. T. \& Alonso, M. M. (2015). Violencia escolar y consumo de alcohol en adolescentes en etapa de secundaria Monterrey, México. Rev enferm Herediana, 8(2), 75-81. Recuperado de https://revistas.upch.edu.pe/index.php/RENH/article/view/2685

Morales, G. F., Cabrera, J. M., Pérez, B. C. \& Amaro, F. M. (2015). El consumo de alcohol en adolescentes de una secundaria de Ciudad Nezahualcóyotl Frecuencia y características sociales. Revista Especializada en Ciencias de la Salud, 18(1), 815. Recuperado de http://www.revistas.unam.mx/index.php/vertientes/article/view/51727/46168

Organización Mundial de la Salud. (2017). Violencia. Recuperado de http://www.who.int/topics/violence/es/

Organización Mundial de la Salud. (2018). Informe Mundial de Situación sobre Alcohol y Salud. Recuperado de http://www.who.int/mediacentre/factsheets/fs349/es/ Polit, D. \& Hungler, B. (2000). Investigación Científica en Ciencias de la Salud (6a ed.). México: Mc Graw-Hill Interamericana.

Pulido I. J. (2014). Consumo de alcohol y violencia escolar en estudiantes de bachillerato, universidad autónoma de Nuevo León. (Tesis de Maestría). Universidad Autónoma de Nuevo León, México Recuperado de http://eprints.uanl.mx/7793/1/1080259474.pdf

Rivero, E.E., Barona, R.C., \& Petriz, M. M. (2011). Violencia escolar en secundarias de México y factores de contexto. Un panorama multivariado desde los exámenes de calidad y logro educativo (Excale). En XI Congreso Nacional de 
Investigación Educativa. Congreso llevado a cabo en Universidad Autónoma del Estado de Morelos, México. Recuperado de http://www.comie.org.mx/congreso/memoriaelectronica/v11/docs/area_17/075 4.pdf

Tegoma, R. V. M., \& Cortaza. R. L. (2016). Prevalencia del consumo de alcohol en adolescentes de una secundaria de Coatzacoalcos, Veracruz. Enfermería universitaria, 13(4): 239-245. https://doi.org/10.1016/j.reu.2016.10.001

Telumbre, J. Y. \& Sánchez, B. E. (2015). Consumo de alcohol en adolescentes del estado de Guerrero, México. Health and Addiction. 15(1), 79-86. Recuperado de https://www.redalyc.org/pdf/839/83938758008.pdf

Todd, L. E. (2010). Adicciones: enfermedades del Siglo XXI. México: Talleres de grafo print.

Ulloa, J. L. (2014). Consumo de alcohol y violencia escolar en estudiantes de bachillerato de tabasco. (Tesis de Maestría). Universidad Autónoma de Nuevo León, México. Recuperado de http://eprints.uanl.mx/4181/1/1080253790.pdf

United Nations Educational, Scientific and Cultural Organization. (2014). Behind the numbers: Ending school violence and bullying. Paris: UNESCO. Recuperado de https://unesdoc.unesco.org/ark:/48223/pf0000366483

Vera, B. D., \& Gálvez, J. L. (2014). Evaluación psicométrica de la escala de conducta delictiva y violenta en el aula, en estudiantes chilenos. Liberabit, 20(2): 325-334. Recuperado de http://www.scielo.org.pe/pdf/liber/v20n2/a13v20n2.pdf

Villareal, M. E. (2009). Un modelo estructural del consumo de drogas y conducta violenta en adolescentes escolarizados (Tesis de Doctorado). Universidad Autónoma de Nuevo León, México. Recuperado de http://eprints.uanl.mx/2080/ 